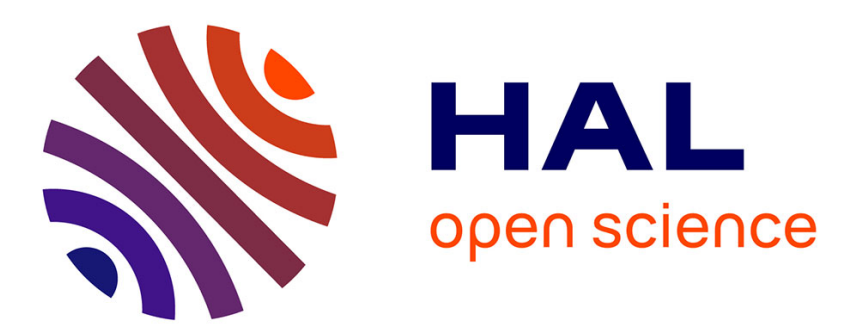

\title{
Hypertensive Disorders of Pregnancy: Kurtosis-Based Classification of Fetal Doppler Ultrasound Signals
}

\author{
Rayan Chaaban, Walaa Issa, Ayache Bouakaz, Amira Zaylaa
}

\section{To cite this version:}

Rayan Chaaban, Walaa Issa, Ayache Bouakaz, Amira Zaylaa. Hypertensive Disorders of Pregnancy: Kurtosis-Based Classification of Fetal Doppler Ultrasound Signals. The 5th International Conference on Advances in Biomedical Engineering, IEEE-EMBS, Oct 2019, Tripoli, Lebanon. inserm-02320950

\section{HAL Id: inserm-02320950 https://www.hal.inserm.fr/inserm-02320950}

Submitted on 19 Oct 2019

HAL is a multi-disciplinary open access archive for the deposit and dissemination of scientific research documents, whether they are published or not. The documents may come from teaching and research institutions in France or abroad, or from public or private research centers.
L'archive ouverte pluridisciplinaire HAL, est destinée au dépôt et à la diffusion de documents scientifiques de niveau recherche, publiés ou non, émanant des établissements d'enseignement et de recherche français ou étrangers, des laboratoires publics ou privés. 


\title{
Hypertensive Disorders of Pregnancy: Kurtosis-Based Classification of Fetal Doppler Ultrasound Signals
}

\author{
Rayan Chaaban ${ }^{1}$, Walaa Issa ${ }^{1}$, Ayache Bouakaz ${ }^{2}$, Amira J. Zaylaa ${ }^{1,3,4 *}$ \\ ${ }^{1}$ Department of Biomedical Engineering, Beirut International University, Beirut, Lebanon \\ ${ }^{2}$ Imaging and Brain Laboratory, Université Frano̧ise Rabelais de Tours, Tours, France \\ ${ }^{3}$ Neoroscience Research Center, Faculty of Medical Sciences, Lebanese University, Lebanon \\ ${ }^{4}$ Faculty of Public Health-V, Lebanese University, Lebanon \\ *Corresponding Author, amira.zaylaa@ul.edu.lb
}

\begin{abstract}
Hypertensive Disorders of Pregnancy (HDP), a group of medical conditions occurring during pregnancy, have wide-reaching implications on the normal progress of $17 \%$ of pregnancies, leading to maternal and perinatal morbidity and mortality. One of the HDP complications is the Intrauterine Growth Restriction (IUGR). IUGR changes the behavior of any feature extracted from Fetal Heart Rates (FHRs). These features if well-selected improve the classification of IUGR. However, the choice of features was reliant on whether it's linear or nonlinear. Also, the classification algorithms such as, K-Means and Support Vector Machine (SVM) used to predict and classify biomedical signals were not optimal, and the best classification algorithm was not yet set. Our aim is to propose a new kurtosisbased combinations of features and explore their effect on HDP and IUGR classification from Doppler Ultrasound FHRs. Features extracted from FHRs were fed into K-means and SVM classification algorithms. The database comprised 50 normal and 50 IUGR FHRs. Results showed that the best extracted features were those based on kurtosis, and the best classification method was the SVM. The best combination result was $67 \%$ sensitive, $100 \%$ specific and $100 \%$ precise to the classification and detection of IUGR and thus HDP. A further future study could test additional combination of features and other classification-based methods to predict IUGR and thus HDP.
\end{abstract}

Index Terms-Hypertensive Disorders of Pregnancy, Intrauterine Growth Restriction, Entropy Features, Kurtosis, K-means clustering, Support Vector Machine Algorithm, Statistical Analysis.

\section{INTRODUCTION}

Hypertensive Disorders of Pregnancy (HDP), also known as maternal hypertensive disorders, is a critical health complications encountering pregnant women and their infants [1]. Such complications affect the normal progress of $17 \%$ of pregnancies [2], leading to Preeclympcia (PE). Early-onset of PE is commonly associated with Intrauterine Growth Restriction (IUGR), an abnormal uterine, and umbilical artery Doppler waveforms [3]. As such, IUGR is one of the major signs and consequences of HDP, also known as Fetal Growth Restriction (FGR), the poor growth of the fetus while in the mother's womb during pregnancy [4]. IUGR is the decreased rate of fetal growth compared to the normal rate of growth.

Many studies have been carried out on feature extraction from Fetal Heart Rates (FHRs), specifically the use of non- linear features, such as entropies to discriminate FHRs [5], [6], [7], [8], [9]. A study published by Zaylaa et al, focused on the employement of Recurrence Plots to promot the discrimination between normal and IUGR fetuses. Entropy and recurrence rates were computed, and the sensitivity, specificity, and accuracy of the recurrence quantification parameters were measured relative to the discrimination of IUGR fetuses [9]. Another study published by Zaylaa et al. provided N-order and maximum fuzzy similarity entropy features for improving the discrimination of FHR signals [7]. Zaylaa et al. focused initially on optimizing the setting parameters for entropy features, and proved that it was advantagous to use the norder similarity entropy over the regular similarity entropy [7]. Another study, explored a cascade of nonlinear kernal-based entropy to discriminate FHRs [10]. Zaylaa et al. presented a statistical evaluation of the discrimination power of each parameter through a paired t-test statistics and distribution spread [10]. However, it was merely applied on brain signals. As FHRs have not only been explored using the theory of complexity [6], [11], [12] or through machine learning and classification. Recently, a crucial study extracted kurtosis feature from cardiac signals and used classification approaches to detect pathologies [13]. However, as per our thorough review, kurtosis is not yet applied to FHRs.

Basically, the aim of the study is to classify HDP through kurtosis extracted from Fetal Doppler Ultrasound information. The database comprised fifty normal pregnancies/FHRs and fifty IUGR pregnancies/IUGR fetuses. IUGR was explored among a set of pathologies in order to reduce its outcomes on the population. Recorded FHRs were collected in the third trimester, to find signatures and to pave the way for a decent and early diagnosis of IUGR associated with HDP. The aim was also to implement and test K-means and SVM algorithms on FHR's features. This includes extracting several kurtosis-based features and classical time-based features, such as Sample Entropy (SampEn) and Fuzzy Entropy (FuzzyEn), from the signals. In addition, choosing new combinations of features, training and testing the algorithms for classification.

The paper is organized as follows. Section I provides 


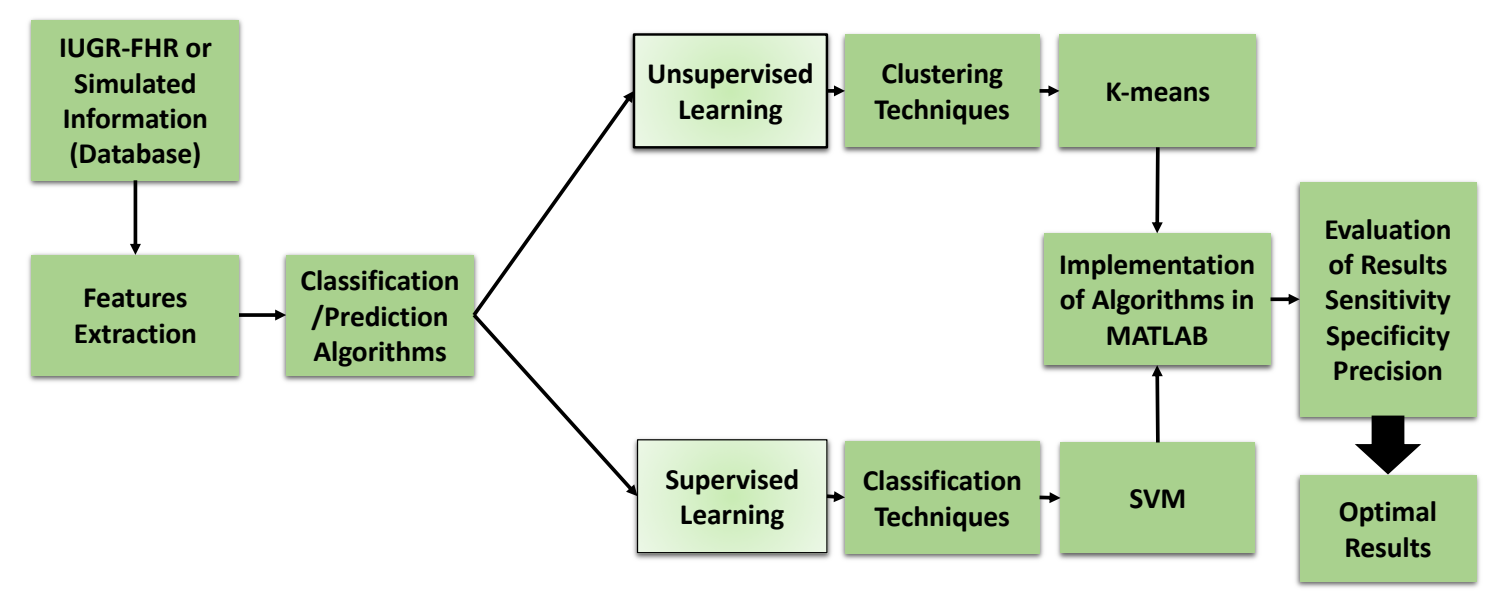

Fig. 1: The Overall Framework of the Study of Hypertensive Disease of Pregnancy (HDP) and Intrauterine Growth Restriction Fetal Heart Rates (IUGR-FHRs) Using Kurtosis-based K-means and Support Vector Machine (SVM) Algorithms.

the literature review and the objectives. Section II presents the materials used for recording FHRs and the processing software. Section III showcases the classical features, the new combinations of kurtosis-based features and the classification methods illustrated in the block diagram in Fig. 1. The block diagram showcases the feature extraction step followed by the classification algorithms applied to FHRs. Section IV showcases the results obtained. Section V provides the discussion and conclusion, and section VI provides the future steps.

\section{MATERIALS}

The Multi-channel Doppler Ultrasound technology (MCDUS) was used for recording FHRs through the Mindray Ultrasound (Mindray North America's Diagnostic Instruments, Inc.). Regular protocols were applied for FHRs extraction [6]. Mindray's Ultrasound (US) is a mobile device comprising three groups of transducers, after some processing steps the extracted clean healthy and IUGR FHRs were displayed on the monitor's screen as shown in Figs. 2 (a) and (b), and were saved. Moreover, the processing was done on MATLAB (R2018a, U.S.).

\section{FEATURES EXTRACTION AND ClASSIFICATION AlgORITHMS OF SignALS}

After loading FHRs, we extracted some features, and then applied the classification techniques. Extracted features were meant to minimize the loss of important information embedded in the signal. The novel part was extracting kurtosis-based combinations of features from FHRs, feeding them to the kmeans and SVM, and evaluating the outcomes statistically.

The features involved included kurtosis and entropy. Entropy, which measures the uncertainty of a system, can be computed in different ways including Sample Entropy (SampEn), Multi-Scale Entropy or Costa Entropy (CostaEn), FuzzyEn, Conditional Entropy (CondEn) and Multi-scale Permutation Entropy (MS-PermEn).
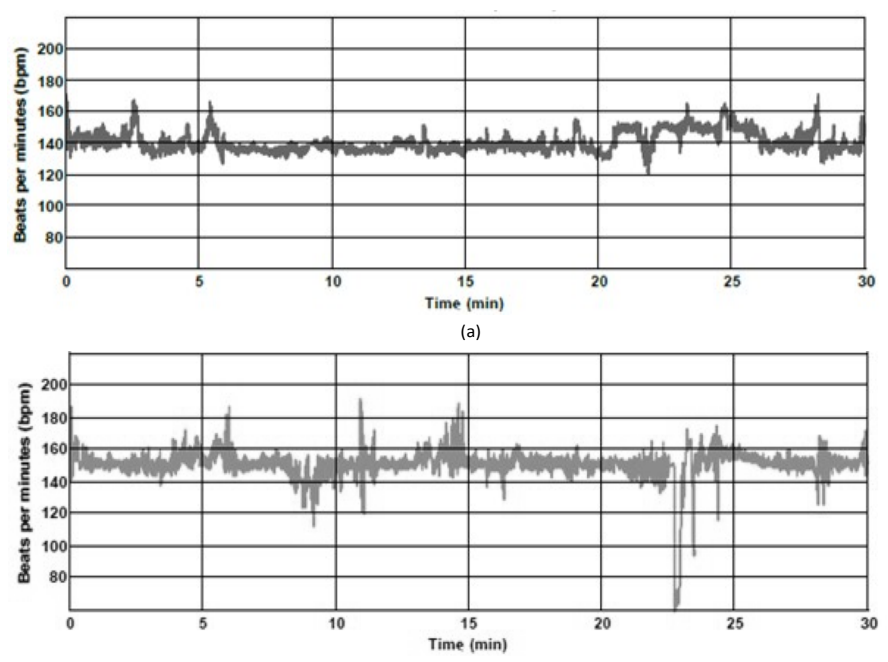

Fig. 2: The Real Fetal Heart Rate Signals. (a) Healthy Fetal Heart Rates (HFHRs). (b) Intrauterine Growth Restricted Fetal Heart Rate (IUGR-FHR).

\section{A. Sample Entropy}

SampEn measures similarity and excludes self-matches from the signal. It is given by:

$$
\operatorname{SampEn}(m . r)=-\ln \frac{A^{m}(r)}{B^{m}(r)},
$$

where $\mathrm{A}$ is the number of pairs of complete matches of length $\mathrm{m}+1$ ( $\mathrm{m}$ being the embedding dimension) placed within a predefined threshold $r=0.2 * s t d($ signal) and $\mathrm{B}$ is the number of pairs of broken matches of length m placed within ' $r$ ' [6].

\section{B. The Multiscale Entropy or Costa Entropy}

MS-En or CostaEn quantifies the complexity of signals by computing the SampEn over a range of scales, i.e. using the coarse-graining procedure to eliminates the fast temporal scales. The new time series $\mathrm{y}(\tau)$ is obtained using the following formula: 
TABLE I: The Selection of the Extracted Feature Sets for the Classification of HDP and IUGR.

\begin{tabular}{|l|l|l|l|l|l|l|}
\hline Features & CostaEn & SampEn & Kurtosis & CondEn & FuzzyEn & MS-PermEn \\
\hline Set I & + & + & - & - & - & - \\
\hline Set II & - & - & + & + & + & - \\
\hline Set III & - & - & + & + & - & + \\
\hline
\end{tabular}

$$
y(\tau)=\frac{1}{\tau} \sum_{i=(j-1) \tau+1}^{j \tau} x_{(i)},
$$

where $\tau$ is the scale factor and for $1 \leq \mathrm{j} \leq \mathrm{N} / \tau$. The length of each coarse-grained time series is $N / \tau$. SampEn provided in section A was then calculated while varying $\tau$ [6].

\section{Fuzzy Entropy}

FuzzyEn measures the quantity of fuzzy information selected from a fuzzy set ' $C$ ' using a membership function, where there are $\mathrm{n}$ membership functions $\mu_{C}$. Assuming that $\mathrm{C}$ is a fuzzy set on discourse $U=\{u 1, u 2, \ldots, u n\}$, the membership vector of $\mathrm{C}$ is $c_{i}=\mu\left(u_{i}\right) \in[0,1]$. FuzzyEn is based on the distance of $\mathrm{C}[6]$ :

$$
H_{\text {FuzzyEn}}(C)=\frac{2}{n} \sum_{i=1}^{n}\left|c_{i}-\mu\left(u_{i}\right)\right| .
$$

where $\mathrm{n}$ is the length of the signal. The outcome is analyzed as follows [6]:

- If $\mathrm{C}$ is a crisp set $\left(c_{i}=0\right.$ or 1$)$, then $H(C)=0$;

- If $c_{i}=\frac{1}{2} \forall i, H(C)$ i.e. $H(C)=1$.

\section{Conditional Entropy}

CondEn quantifies the amount of information needed to describe the outcome of a discrete and random signal $\mathrm{Y}$ given another signal $\mathrm{X}$ [14]. The entropy of $\mathrm{Y}$ conditioned on $\mathrm{X}$ is written as $H(Y / X)$. If $H(Y / X=x)$, CondEn is defined by [14]:

$$
H_{\text {CondEn }}=\sum_{x \in X} P(x) H\left(\frac{Y}{X}=x\right) .
$$

where $P(x)$ is the probability function of $\mathrm{x}$.

\section{E. Multi-scale Permutation Entropy}

PermEn algorithm splits the data into overlapping n-tuples patterns, where $\mathrm{n}$ is the embedding dimension. Each $\mathrm{n}$-tuple is then sorted in ascending order, which generates a permutation type $\pi$ according to the ordering of the sorted data. For a signal $x_{0}, \ldots, x_{N-1}$ PermEn(n) is calculated as follows:

$$
\operatorname{PermEn}(n)=-\sum_{i}^{n !} P\left(\pi_{i}\right) \log P\left(\pi_{i}\right),
$$

where $P\left(\pi_{i}\right)$ is the number of occurrence. PermEn(n) ranges from 0 to $\log (\mathrm{n}$ !), with 0 indicating a series that is monotonically increasing or decreasing (highly regular), and $\log (n$ !) indicates a completely random series [15]. MS-PermEn is the PermEn applied to the coarse grained signals.

\section{F. Kurtosis}

Kurtosis is the measure of the thickness or heaviness of the tails of a distribution of the signal, and the measure of the "tailedness" of the probability distribution [13]. Kurtosis is a descriptor of the shape of a probability distribution. There are various interpretations of the degree of excess kurtosis:

- low for flat frequency distribution

- high for peaked frequency distribution [13].

Higher kurtosis is the result of infrequent extreme deviations as opposed to frequent modestly sized deviations. The excess kurtosis is given by:

$$
\operatorname{Kurt}(Y)-3=\frac{1}{\left(\sum_{j=1}^{n} \sigma_{j}^{2}\right)^{2}} X \sum_{i=1}^{n} \sigma_{i}^{4}\left(\operatorname{Kurt}\left(X_{i}\right)-3\right) .
$$

where $\sigma_{i}$ the standard deviation of $X_{i}, Y$ is a random signal defined by the sum of the $X_{i}$ (here FHR signals) and kurt is given as: $\operatorname{Kurt}(X)=\frac{\mu_{4}}{\sigma^{4}}$, where $\mu_{4}$ is the fourth central momentum.

In our study, the features were divided into three sets as shown in Table I. Set I, [CostaEn, SampEn], which was based on various complexity analysis studies [5], [6], [7], and both Set II, [Kurtosis, CondEn, FuzzyEn], and Set III, [Kurtosis, CondEn, MS-PermEn], which were the new kurtosis-based combination of features.

\section{G. Signals Classification and Clustering}

Each of the three sets of features was fed into two classification techniques, K-Means and SVM. The classification approach was used to assign different FHR signals to different groups, or to partition a data set into clusters, so that the data in each subset shares some common trait proximity, according to a predefined distance measure [16]. K-means and SVM are considered as supervised and non-supervised clustering techniques, respectively [16]. The final step demonstrated in Fig. 1 is based on the evaluation of the classification and detection of IUGR and thus HDP through the sensitivity, specificity and precision measures in percentage.

\section{Simulation Results}

According to the features extracted, there are differences in the outcomes of the classification and clustering results of normal and the IUGR signals. The sensitivity, specificity and precision percentages were calculated as in [9], and are shown in Table II. The highest statistical values were highlighted in bold.

The [Kurtosis, CondEn, FuzzyEn] combination of features was $100 \%$ sensitive, $38 \%$ specific and $55 \%$ precise to the classification and detection of IUGR and HDP using k-means method, while [CostaEn, SampEn] showed lower percentages and lower significance using K-means (Table II).

Set III was $100 \%$ sensitive, $75 \%$ specific and $80 \%$ precise in the classification of FHRs. Moreover, set II and set III were 
TABLE II: The Statistical Evaluation of the Classification of IUGR and HDP through three Different Combinations of Features Extracted from Fetal Heart Rates Using the K-means and the Support Vector Machine (SVM).

\begin{tabular}{|c|c|c|c|c|}
\hline Studies & Classification/Clustering Algorithms & Sensitivity $(\%)$ & Specificity $(\%)$ & Precision $(\%)$ \\
\hline \multirow[t]{2}{*}{ Zaylaa et al. ${ }^{[6,7]}$ and Costa et al. ${ }^{[7]}$ (Set I) } & K-Means & 100 & 33 & 50 \\
\hline & SVM & 100 & 67 & 80 \\
\hline \multirow[t]{2}{*}{ New Combination (Set II) } & K-Means* & 100 & 38 & 55 \\
\hline & SVM & 83.3 & 67 & 83 \\
\hline \multirow[t]{2}{*}{ New Combination (Set III) } & K-Means* & 100 & 75 & 80 \\
\hline & SVM $^{*}$ & 67 & 100 & 100 \\
\hline
\end{tabular}

highly specific and precise to the classification as compared to set I using the SVM algorithm.

The classification of IUGR and HDP mostly significant using the kurtosis-based combination of features, i.e. [Kurtosis, CondEn, MS-PermEn] followed by [Kurtosis, CondEn, FuzzyEn] features using SVM, as opposed to the classical [CostaEn, SampEn] feature.

\section{Discussion AND CONCLUSION}

Our results revealed that if the number of features in SVM increases, the specificity incearses by $16.5 \%$ and the precision of the classification increases by $11.5 \%$ as compared to the reference results obtained by [CostaEn, SampEn] features [5], [6], [7], however the sensitivity decreases. This decrase in sensitivity supports the findings of Al-Angari et al. [13] which showed that such types of kurtosis-based features are not only sensitive to detect IUGR but also sensitive to cardiac diseases. Kurtosis results were precise (55-100\%) which is in agreement with the work done by Fan and Zuo [17].

Our work shows the important steps of extracting kurtosisbased features, as they served as signatures for the change in the state of the Doppler Ultrasound FHR information. Among the classification methods SVM was advantageous over the K-means. The study explores the problem of IUGR caused by the HDP using the classification of real FHRs. FuzzyEn features existing in literature and classification algorithms were implemented. Real results obtained could be useful to promote the classification and reduce the percentage and complications of HDP on pregnancies.

\section{FUTURE WORK}

Our exploratory study included the k-means and SVM to perform the classification of IUGR and thus HDP through the new kurtosis-based combination of features. As a prospect, there are various different tests of new features and experiments are yet to be implemented.

\section{REFERENCES}

[1] L. Zhenhu, D. Xuejing, and L. Xiaoli, Entropy Measures in Neural Signals. Singapore: Springer Singapore, 2016, pp. 125-166. [Online]. Available: https://doi.org/10.1007/978-981-10-1822-0_8

[2] S. Singh, E. B. Ahmed, and S. Constan, NICP," PMC, [Online], SepOct 2014.
[3] D. J. Sawchuck and B. K. Wittmann, "Pre-eclampsia renamed and reframed: Intra-abdominal hypertension in pregnancy," Medical Hypotheses, vol. 83, no. 5, pp. 619 - 632, 2014. [Online]. Available: http://www.sciencedirect.com/science/article/pii/S0306987714002722

[4] G. Mandruzzato, A. Antsaklis, F. Botet, and et al., "Intrauterine restriction (iugr)," Journal of Perinatal Medicine, vol. 36, no. 4, p. 277281 , 2008.

[5] S. Oudjemia, A. Zaylaa, J. Charara, and J.-M. Girault, "Delta-fuzzy similarity entropy to discriminate healthy from sick fetus," in Proc. ICABME. IEEE, 2013, pp. 1-4.

[6] A. Zaylaa, "Analysis and extraction of complexity parameters of biomedical signals," Ph.D. dissertation, François-Rabelais University of Tours, 2014.

[7] A. Zaylaa, S. Oudjemia, J. Charara, and J.-M. Girault, "n-order and maximum fuzzy similarity entropy for discrimination of signals of different complexity: Application to fetal heart rate signals," Computers in biology and medicine, vol. 64, pp. 323-333, 2015.

[8] A. Zaylaa, A. Harb, F. Khatib, Z. Nahas, and F. Karameh, "Entropy complexity analysis of electroencephalographic signals during pre-ictal, seizure and post-ictal brain events," in Proc. ICABME,. IEEE, 2015, pp. $134-137$.

[9] A. Zaylaa, J. Charara, and J. Girault, "Advanced discrimination between healthy and intrauterine growth restricted fetuses by unbiased recurrence plots," Advanced Techniques in Biology and Medicine, vol. 4, no. 2, pp. 1-10, 2016.

[10] A. Zaylaa, S. Saleh, F. Karameh, Z. Nahas, and A. Bouakaz, "Cascade of nonlinear entropy and statistics to discriminate fetal heart rates," in Advances in Computational Tools for Engineering Applications (ACTEA), 2016 3rd International Conference on. IEEE, 2016, pp. 152-157.

[11] A. Zaylaa, J.-M. Girault, and J. Charara, "Unbiased recurrence plot quantification of chaotic dynamic systems by eliminating sojourn points," in Proc. ICABME. IEEE, 2013, pp. 187-190.

[12] A. Zaylaa, J. Charara, and J.-M. Girault, "Reducing sojourn points from recurrence plots to improve transition detection: Application to fetal heart rate transitions," Computers in biology and medicine, vol. 63, pp. 251-260, 2015.

[13] H. M. Al-Angari, Y. Kimura, L. J. Hadjileontiadis, and A. H. Khandoker, "A hybrid emd-kurtosis method for estimating fetal heart rate from continuous doppler signals," Frontiers in physiology, vol. 8, p. 641, 2017.

[14] P. Gaspard, Rossler System. Encyclopedia of Nonlinear Science, 2005, no. $808-811$.

[15] F. C. Morabito, D. Labate, F. La Foresta, A. Bramanti, G. Morabito, and I. Palamara, "Multivariate multi-scale permutation entropy for complexity analysis of alzheimers disease eeg," Entropy, vol. 14, no. 7, pp. 1186-1202, 2012.

[16] S. Al Obaidly and A. Kurjak, "Prenatal diagnosis of morbidly adherent placenta with $2 \mathrm{~d}$ ultrasonography, $3 \mathrm{~d}$ color power doppler and magnetic resonance imaging," Donald School Journal of Ultrasound in Obstetrics and Gynecology, vol. 4, no. 2, pp. 199-204, 2010.

[17] F. Xianfeng and J. Z. Ming, "Machine fault feature extraction based on intrinsic mode functions," Measurement Science and Technology, vol. 19, no. 4, p. 045105, feb 2008. [Online]. Available: https://doi.org/10.1088\%2F0957-0233\%2F19\%2F4\%2F045105 